\title{
A Self-Consistent Model of Helium in the Thermosphere
}

Eric K. Sutton, ${ }^{1}$ Jeffrey P. Thayer, ${ }^{2}$ Wenbin Wang, ${ }^{3}$ Stanley C. Solomon, ${ }^{3}$

Xianjing Liu, ${ }^{2,4}$ and Benjamin T. Foster ${ }^{3}$

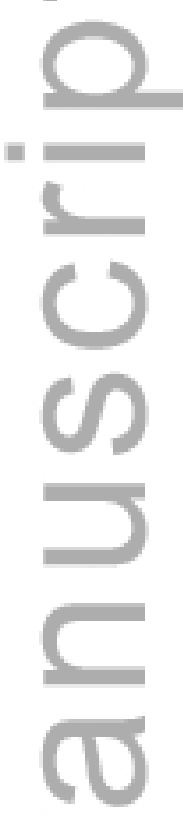

E. K. Sutton, Space Weather Center of Excellence, Air Force Research Laboratory, Albuquerque, NM 87117, USA. (eric.k.sutton@gmail.com)

${ }^{1}$ Space Weather Center of Excellence, Air

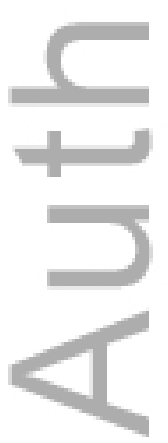

This is the author manuscript accepted for publication and has undergone full peer review but has not been through the copyediting, typesetting, pagination and proofreading process, which may lead to differences between this version and the Version of Record. Please cite this article as doi:
D R0A10五2 2 /2015JA021223
August 10, 2015, 8:56pm
D R A F T

This article is protected by copyright. All rights reserved. 
3 Abstract. We have found that consideration of neutral helium as a ma-

4 jor species leads to a more complete physics-based modeling description of

5 the Earth's upper thermosphere. An augmented version of the composition

${ }_{6}$ equation employed by the Thermosphere-Ionosphere-Electrodynamic Gen-

7 eral Circulation Model (TIE-GCM) is presented, enabling the inclusion of

s helium as the fourth major neutral constituent. Exospheric transport act-

9 ing above the upper boundary of the model is considered, further improv-

10 ing the local time and latitudinal distributions of helium. The new model

${ }_{11}$ successfully simulates a previously observed phenomenon known as the "win-

Force Research Laboratory, Albuquerque,

New Mexico, USA.

${ }^{2}$ Aerospace Engineering Sciences

Department, University of Colorado,

Boulder, CO, USA.

${ }^{3}$ High Altitude Observatory, National

Center for Atmospheric Research, Boulder,

Colorado, USA

${ }^{4}$ present affiliation: Department of

Atmosphere, Oceanic and Space Sciences,

University of Michigan, Ann Arbor, MI,

USA. 
${ }_{12}$ ter helium bulge," yielding behavior very similar to that of an empirical model

13 based on mass spectrometer observations. This inclusion has direct conse-

14 quence on the study of atmospheric drag for low-Earth orbiting satellites,

15 as well as potential implications on exospheric and topside ionospheric re-

${ }_{16}$ search.
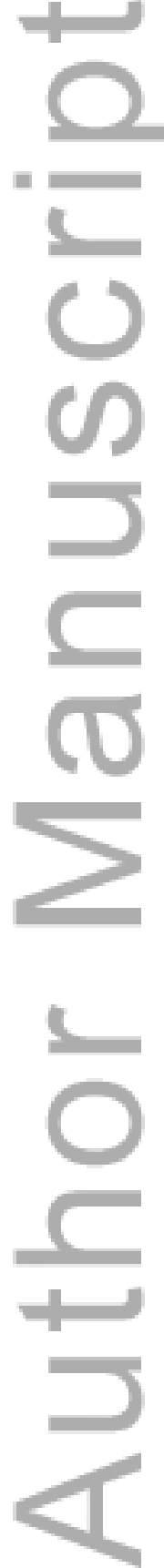


\section{Introduction}


38 assumption of diffusive equilibrium to separate composition-induced mass density varia-

39 tions from those caused by temperature. In response, Keating et al. [1974] augmented

40 their drag-inferred technique to include a description of the background composition that

${ }_{41}$ was consistent with the available mass spectrometer data. New ratios in excess of an order

42 of magnitude could then be obtained through this method as well. In addition to estab-

43 lishing a larger bulge ratio, Reber et al. [1971] noted a strong correlation of the maximum

${ }_{44}$ helium density with the location of the winter geomagnetic pole. This was interpreted as

${ }_{45}$ a sensitivity of the helium distribution to the thermospheric wind system.

${ }_{46}$ In addition to high-latitude variations near the solstices, Newton et al. [1973] detected a ${ }_{47}$ strong local time preference for helium concentration as measured by mass spectrometers 48 on the low-inclination San Marco 3 satellite. Reber et al. [1973] and Mayr et al. [1974]

49 discussed similar variations manifest within the Ogo 6 density model [Hedin et al., 1974].

50 These findings showed a preference of the diurnal maxima toward earlier times for species ${ }_{51}$ with small molecular masses, with the opposite being true for species of large mass. The ${ }_{52}$ San Marco 3 observations, taken at altitudes near $225 \mathrm{~km}$, showed a preference toward ${ }_{53}$ the 06-09 LT sector while those taken by Ogo 6, near $450 \mathrm{~km}$, showed maxima closer to

${ }_{54} 10 \mathrm{LT}$.

${ }_{55}$ The realization of these phenomena motivated several modeling studies to uncover the ${ }_{56}$ mechanism responsible for the counterintuitive distribution of helium in the thermosphere.

${ }_{57}$ Noticing that helium vertical profiles measured by several rocket-based mass spectrometers

${ }_{58}$ departed quite drastically from diffusive equilibrium, an early study by Kasprzak [1969] ${ }_{59}$ invoked an additional diffusive flux in order to reconcile the observations with his model.

${ }_{60}$ This treatment required vertical fluxes on the order of $6 \times 10^{8}$ and $2 \times 10^{10} \mathrm{~cm}^{-2} \mathrm{~s}^{-1}$ 
${ }_{61}$ for summer and winter conditions, respectively, over an altitude range of 120-200 km.

${ }_{62}$ Kockarts [1973] later noted, however, that these values were larger than the maximum

${ }_{63}$ flux allowed by molecular diffusion, thus requiring an additional mechanism of transport.

${ }_{64}$ Johnson and Gottlieb [1970] used basic considerations of continuity to show that a 65 general summer-to-winter flow of the major atmospheric constituents could account for ${ }_{6}$ a buildup of helium in the winter polar regions. Without discounting these findings, ${ }_{67}$ several attempts were made to ascertain the effect of atmospheric fluctuations on helium ${ }_{68}$ transport. Hodges [1970] modeled large-scale fluctuations as monochromatic plane waves,

${ }_{69}$ which effected a downward transport and an overall decrease to the scale height of species 70 with masses smaller than the mean mass. Similarly, Kockarts [1972] derived the eddy

${ }_{71}$ diffusivity profile necessary to reconstruct the winter helium bulge observations of Reber ${ }_{72}$ et al. [1971], under the assumption of molecular diffusion in the absence of wind. Results ${ }_{73}$ from these studies suggested that eddy diffusion could in fact control the global helium ${ }_{74}$ distribution. However, recreating the observed winter bulge ratios required more than 75 an order-of-magnitude increase in eddy diffusivity from winter to summer hemispheres.

76 These results were qualitatively consistent with each other, yet they implied that similar

77 latitudinal signatures should be evident in other minor atmospheric constituents, a feature 78 that was inconsistent with previous observations of atomic oxygen [Kockarts, 1973].

79 Reber and Hays [1973] performed a more rigorous treatment of the effects of circulation ${ }_{80}$ on the distribution of helium. Included in their model were the effects of molecular and ${ }_{81}$ eddy diffusion as well as a parameterized circulation pattern of the background gas that 82 satisfied continuity requirements and could be tuned to simulate a given level of summersз to-winter flow. Combining the equations of continuity and momentum for a minor species 
${ }_{84}$ led to an accurate representation of previous winter helium bulge observations. The idea that the winter helium bulge could be completely explained by seasonal circulation pat-

${ }_{86}$ terns led, however, to an apparent paradox. At times of high solar flux, when an enhanced

${ }_{87}$ summer-to-winter flow had been expected to occur, smaller pole-to-pole helium ratios had

s been observed. Reber and Hays [1973] explained the discrepancy by invoking the mecha-

${ }_{89}$ nism of exospheric flow, whereby during times of high solar flux, increased temperatures

${ }_{90}$ in the upper thermosphere drive a larger exospheric flow directed away from the winter

${ }_{91}$ bulge. The balance between the circulation-induced effects and exospheric transport was

${ }_{92}$ found to control the magnitude of the latitudinal gradient in helium concentration that

${ }_{93}$ could be supported by the atmosphere.

94 By analyzing the combined equations of continuity and momentum for a minor species,

${ }_{95}$ Reber and Hays [1973] and Hays et al. [1973] identified the vertical advection term as

${ }_{96}$ being responsible for establishing the seasonal distribution of helium. In the presence ${ }_{97}$ of diffusively separated atmospheric constituents, this term leads to increased helium densities in regions of downwelling and decreased densities in regions of upwelling. The opposite behavior is implied for species, such as argon, that are heavier than the local 
${ }_{107}$ diffusively separated constituents. Both groups agreed that the interaction between he${ }_{108}$ lium and the background circulation - consisting of upwelling in the summer hemisphere,

109

110

111 
151 taken into account by applying seasonal variation of the eddy diffusivity coefficient at ${ }_{152}$ the lower boundary [Qian et al., 2009, 2013]. Based on measurements from the Mauna ${ }_{153}$ Loa Observatory [Keeling and Whorf, 2005], the mixing ratio of $\mathrm{CO}_{2}$ imposed at the ${ }_{154}$ lower boundary was set to 364 ppmv for 1996, increasing linearly by 1.5 ppmv per year thereaftēr.

In the simulations presented throughout this paper, solar irradiance is specified in a manner consistent with Solomon et al. [2011]. The $\mathrm{M}_{10.7}$ index is used in place of the $\mathrm{F}_{10.7}$ solar proxy in an effort to better capture solar UV and EUV irradiance during the deep solar minimum of 2008 . The $\mathrm{M}_{10.7}$ index derives from the magnesium core-to-wing (MgII c/w) of Viereck et al. [2004] via a linear fit to the $\mathrm{F}_{10.7}$ proxy calculated during 1978-2007 [Solomon et al., 2011]. With this normalization, $\mathrm{M}_{10.7}$ can be used in place of $\mathrm{F}_{10.7}$ to drive the EUVAC proxy model [see Richards et al., 1994; Woods and Rottman, 2002; Solomon and Qian, 2005].

Magnetospheric inputs to the polar regions are specified by an applied electric potential pattern and an auroral precipitation oval. The Heelis et al. [1982] empirical specification of magnetospheric potential in the ionosphere, which is parameterized by the 3-hour geomagnetic $\mathrm{K}_{\mathrm{P}}$ index, is the standard TIE-GCM input and is employed for the simulations presented throughout this paper. Auroral precipitation is applied as described by Roble and Ridley [1987] based on the estimated hemispheric power of precipitating electrons.

The empirical estimate of this power as it depends on $\mathrm{K}_{\mathrm{P}}$ has been increased from its original formulation by a factor of $\sim 2$, based on results from the Global Ultraviolet Imager (GUVI) on the TIMED satellite [Zhang and Paxton, 2008]. 
179 of model uncertainties.

\subsection{Helium as a Major Species}


${ }_{218}$ perhaps the dominant component - under certain conditions within the spatial domain of

225 variables)

$$
L_{\ddot{u}}=\frac{\partial}{\partial z}-\left(1-\frac{m_{i}}{\bar{m}}-\frac{1}{\bar{m}} \frac{\partial \bar{m}}{\partial z}-\frac{\alpha_{T i}}{T} \frac{\partial T}{\partial z}\right)
$$

The meanings of several variables have been modified from those originally intended by Dickinson et al. [1984]. $\Psi$ is now the vector of mass mixing ratios for $\mathrm{O}_{2}, \mathrm{O}$, and $\mathrm{He}$, while the mass mixing ratio of the remaining major constituent $\mathrm{N}_{2}$ is specified by $\psi_{N_{2}}=$ $1-\psi_{\mathrm{O}_{2}}-\psi_{\mathrm{O}}-\psi_{\mathrm{He}}$. Molecular and thermal diffusion are accounted for by the first term on the right side of Eq. (1), eddy diffusion by the second, horizontal and vertical advection by the third, and chemical sources and sinks by the fourth.

$\mathbf{L}$ is a diagonal matrix operator with elements:

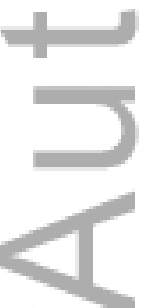

which have been expanded to describe thermal diffusion, a phenomenon which becomes important for species such as helium whose masses are quite different from the mean mass. 
${ }_{235}$ We use a simplified formulation of thermal diffusion that is analogous to its appearance in ${ }_{236}$ the binary diffusion equations, after Colegrove et al. [1966]. In this treatment, a constant ${ }_{237}$ value of $\alpha_{H e}=-0.38$ is used. While this value is characteristic of small concentrations ${ }_{238}$ of helium diffusing through molecular nitrogen, this assumption is reasonably accurate at ${ }_{239}$ altitudes where significant temperature gradients exist (i.e. below $\sim 200 \mathrm{~km}$ ) [Banks and ${ }_{240} \quad$ Kockarts, 1973].

The normalized molecular diffusion matrix, $\boldsymbol{\alpha}$, couples the major components to one another. As can be seen in Eqs. (A18) and (A23) in the appendix, the strength of this ${ }_{243}$ coupling depends on the mutual diffusion coefficients. Dickinson et al. [1984] assumed ${ }_{244}$ these coefficients to take the form $D=D_{0}\left(T / T_{00}\right)^{1.75}\left(p_{00} / p\right)$ for the major species, after ${ }_{245}$ Colegrove et al. [1966]. Accordingly, the elements of $\boldsymbol{\alpha}$ have been normalized by this ${ }_{246}$ functional form. Mutual diffusion coefficients between helium and the other three major ${ }_{247}$ species take a similar form, yet with exponents, $s$, that deviate slightly from 1.75 , as seen ${ }_{248}$ in Table (2.2). These differences have been accounted for by applying correction factors ${ }_{249}$ of the form $\left(T / T_{00}\right)^{1.75-s}$ to the appropriate terms within the diffusion matrix $\boldsymbol{\alpha}$. In ${ }_{250}$ the absence of these corrections, the coefficient describing the mutual diffusion between ${ }_{251}$ helium and atomic oxygen would remain reasonably accurate, yet those describing the ${ }_{252}$ interaction of helium with molecular species would be approximately $5 \%$ low.

The chemical source and sink matrix, s, also serves to couple the major species to one another. In the case of helium, however, all chemical and photochemical rates have been ${ }_{255}$ set to zero, consistent with our assumption of inertness. Therefore, our current model ${ }_{256}$ implementation is appropriate for the study of the dynamical behavior of helium as an ${ }_{257}$ ideal inert tracer. 
The neutral thermodynamic properties of specific heat, $c_{p}$, molecular viscosity, $k_{m}$, and conductivity, $k_{t}$, have been augmented to include the effects of helium. The following 260 equations are now used [Banks and Kockarts, 1973]:

$$
\begin{aligned}
& c_{p}=\frac{R}{2 n}\left(\frac{7}{32} n_{O_{2}}+\frac{5}{16} n_{O}+\frac{7}{28} n_{N_{2}}+\frac{5}{4} n_{H e}\right) \mathrm{erg} \cdot \mathrm{g}^{-1} \mathrm{~K}^{-1} \\
& k_{m}=\frac{10^{-6} T^{0.69}}{n}\left(4.03 n_{O_{2}}+3.90 n_{O}+3.43 n_{N_{2}}+3.84 n_{H e}\right) \mathrm{g} \cdot \mathrm{cm}^{-1} \mathrm{~s}^{-1} \\
& k_{t}=\frac{T^{0.69}}{n}\left(56.0\left(n_{O_{2}}+n_{N_{2}}\right)+75.9 n_{O}+299.0 n_{H e}\right) \mathrm{erg} \cdot \mathrm{cm}^{-1} \mathrm{~s}^{-1} \mathrm{~K}^{-1}
\end{aligned}
$$

261

\subsection{Boundary Conditions}

At the lower boundary of the model, atomic and molecular oxygen adhere to the conditions specified in the original TIE-GCM implementation, namely, that the peak of the atomic oxygen density profile lies at the lower boundary and the total amount of oxygen atoms remains constant making up $23.4 \%$ of the total mass. In addition, we specify a constant lower boundary mass mixing ratio for helium of $1.154 \times 10^{-6}$. In terms of mass 
${ }_{284}$ a vertical outward particle flux:

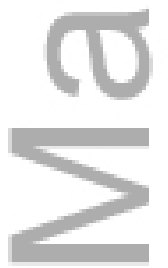

$$
\Phi=-\nabla^{2}\left(n \bar{v} H^{2} P\right)
$$

${ }_{285}$ where $\nabla^{2}$ is the surface Laplacian. The variables $\Phi, n, \bar{v}$, and $H$ are respectively the vertical particle flux, number density, mean thermal speed, and scale height, all specific to

${ }_{287}$ helium. $P$, a dimensionless factor arising from integration over Maxwellian distributions, ${ }_{288}$ has a weak dependence on neutral temperature that can be adequately approximated by 289 [Hodges and Johnson, 1968]:

$$
P \approx\left(1+\frac{T}{3300}\right)
$$


${ }_{290}$ for neutral temperature, $T$, in units of Kelvin. Inherent in these equations is the assump-

${ }_{291}$ tion that collisions do not occur above the upper boundary of the TIE-GCM.

In practice, this vertical flux can be prescribed at the upper boundary of the model as

${ }_{293}$ a diffusive flow. The following vector equation describing molecular diffusion is used:

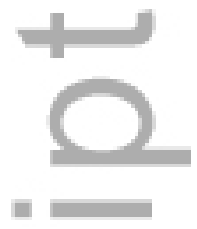

$$
\mathbf{w}_{\mathbf{D}}=\tau^{-1}\left(\frac{T_{00}}{T}\right)^{0.25} \frac{p_{0} \bar{m}}{g m_{N_{2}}} \boldsymbol{\alpha}^{-1} \mathbf{L} \boldsymbol{\Psi}
$$

${ }_{294}$ where $\mathbf{w}_{\mathbf{D}}$ is the $(3 \times 1)$ vector of vertical diffusive mass flow rates for $\mathrm{O}_{2}, \mathrm{O}$, and He,

295 respectively. From the derivation of Eq. (9) in the appendix (see Eq. A25), it follows that

${ }_{296}$ the diffusive mass fluxes of all neutral species sum to zero. Because molecular oxygen and nitrogen densities are small near the upper boundary, we enforce this constraint

${ }_{298}$ by assuming that any outward (inward) mass flux of helium is balanced by an inward

299 (outward) flux of atomic oxygen. Any error that this assumption incurs in the solution of 300 atomic oxygen concentration is diminished by the factor of 4 difference between the mass 301 of oxygen and helium atoms.

302 In the current implementation of our model, the argument of the Laplacian from Eq. (7) ${ }_{303}$ is transformed into a non-aliasing spherical harmonic expansion. This is completed using ${ }_{304}$ the technique of Swarztrauber [1979], modified to accommodate the TIE-GCM's horizontal grid which is offset from the pole by a half-grid increment. The flux, $\Phi$, is then calculated using the well-known eigenfunction/eigenvalue relation:

$$
\nabla^{2} Y_{n}^{m}=-\frac{n(n+1)}{R^{2}} Y_{n}^{m}
$$


${ }_{307}$ where $Y_{n}^{m}$ refers to the spherical harmonic function of degree $n$ and order $m$, and $R$ is ${ }_{308}$ a characteristic radius of the exobase. In the current implementation, $R$ has been set to ${ }_{309}$ the radius of the Earth for consistency with calculations of other horizontal derivatives ${ }_{310}$ within the TIE-GCM. The mass flux required by the left-hand-side of Eq. (9) can then be 311 obtained by transforming back to the spherical grid and multiplying the obtained particle ${ }_{312}$ flux by the molecular mass of helium. The advantage of using this technique in place of ${ }_{313}$ finite differences for calculating the Laplacian is that waves are resolved uniformly over 314 the Earth. Therefore, the growth of numerical instabilities can be controlled by truncating 315 the expansion prior to transforming back to the spherical grid. We note that the degree 316 of truncation required is sensitive to the level of the upper boundary, the grid-size, and 317 the time step. When using the default $5^{\circ} \times 5^{\circ} \times H / 2$ spatial grid with upper boundary of ${ }_{318} z=+7$ and a 120 second time step, we have found that a triangular truncation of degrees 319 higher than 4 is sufficient to limit the growth of numerical instabilities without severely ${ }_{320}$ compromising the accuracy of the exospheric transport model. The adjustment of this ${ }_{321}$ truncation parameter, as well as the characteristic exobase radius, $R$, are left as tasks for 322 future work.

\section{Global Features}

323

324
August 10, 2015, 8:56pm

D R A F T 
${ }_{329}$ to aid in visualization, specifically regarding the sensitivity of the helium distribution to

Figure 2 shows helium densities at $250 \mathrm{~km}$ altitude simulated by the TIE-GCM during each of the four seasons of 2008 . The winter helium bulge phenomenon is clearly present at both solstices. During the equinoxes, the helium bulge undergoes a migration from the spring hemisphere to the fall. Along the way, helium levels are briefly enhanced at low latitudes with a strong preference for early morning local times, with the full transistion taking approximately 1-2 months. At winter solstice, a similar preference for early morning is tempered by an aversion to the auroral zones, where pockets of divergence and upwelling lead to localized helium depletions. This balance manifests as a diurnal modulation of the winter helium bulge in latitude and local time. Symptoms of this behavior can be seen in the the upper right panel of Figure 2, where the southern hemisphere winter peak occurs around 16:00 LT. For reference, the geomagnetic poles are located at $82.4^{\circ} \mathrm{N} / 18: 30 \mathrm{LT}$ and $74.5^{\circ} \mathrm{S} / 8: 20 \mathrm{LT}$ in these plots. Movie S1 also captures this diurnal undulation and its relationship with the distribution of auroral heating during southern hemisphere winter. Constant solar and geomagnetic forcing parameters were used to create the one-day looping animation.

The high-latitude helium distribution is further complicated by short-scale variations in geomagnetic heating. In general, helium densities tend to increase at low latitudes during periods of geomagnetic activity. The opposite is true in the polar region during solstice, as the high-latitude upwelling and divergence resulting from geomagnetic activity tend to so lift heavy constituents while dispersing helium over a larger horizontal expanse. 
${ }_{374}$ helium distributions clearly exhibit the same strong preference for the winter polar regions

375 during solstice, and for the low-latitude, early local time sectors during equinox. Likewise,

${ }_{376}$ a similar sensitivity to geomagnetic effects is evident within MSIS. Notice, however, that

${ }_{377}$ the color scales differ between Figures 2 and 3 in order to show behavior over the full

${ }_{378}$ range of each model. At $250 \mathrm{~km}$, the TIE-GCM typically underestimates the magnitude

${ }_{379}$ of the MSIS helium bulge by approximately $20 \%$ during solstice, while overestimating it

380 by $5 \%$ during equinox. This agreement is reasonable, considering that no adjustments

${ }_{381}$ have been made to the TIE-GCM in an effort to improve model agreement. Likewise, the

382 MSIS model estimated and applied correction factors for the underlying mass spectrom-

звз eter data [Hedin, 1987], which could further limit the absolute accuracy of such model

${ }_{384}$ comparisons. In certain cases, there are discrepancies in the location and shape of the

${ }_{385}$ helium bulge between models. For instance, the location of maximum helium concentra-

386 tion during the June solstice is out of phase by about 8 hours in local time between the

387 two models. While the MSIS helium distribution is prescribed, to a certain extent, by a

388 trade-off between the data sparsity of its underlying historical data set and the complexity

389 of its basis functions, further investigation is needed before attributing any discrepancies

390 to the shortcomings of either model.

Figure 4 shows the magnitude of the helium bulge ratio as a function of height, during 
${ }_{397}$ profiles exhibit a quick increase from the lower boundary, giving way to a maximum ${ }_{398}$ around $175 \mathrm{~km}$, then decaying slowly with altitude to the upper boundary. This behavior 399 can be explained by the transition from a region below the peak which is dominated by 400 collisions, to a region above the peak where diffusive equilibrium is well established. Below ${ }_{401}$ the height of maximum bulge ratio, the summer-to-winter bulk circulation pattern leads 402 to the accumulation of helium in the winter hemisphere. Above this height, however, ${ }_{403}$ vertical profiles begin to approximate diffusive equilibrium, causing helium densities in ${ }_{404}$ the winter hemisphere to decrease with height at a slightly faster exponential rate than ${ }_{405}$ those in the warmer summer hemisphere.

The significant difference between June and December is due to a combination of lower solar flux and geomagnetic activity during the December solstice. Smaller contributions ${ }_{408}$ to this difference may arise from seasonal variations such as in the eddy diffusivity. Error ${ }_{409}$ bars in Figure 4 show the standard deviation of the helium ratio over the course of a day, ${ }_{410}$ giving an indication of the sensitivity to diurnal variations as well as small variations in ${ }_{412}$ variations in the lower part of the profile take place on longer timescales. Presumably, the ${ }_{413}$ lower portion of the profile is more sensitive to season and solar flux than to short-scale ${ }_{414}$ geomagnetic activity. Approaching altitudes as low as $100 \mathrm{~km}$, the two profiles begin ${ }_{415}$ to converge, suggesting a muted response to geomagnetic activity as well as to seasonal 416 variations,

${ }_{417}$ The addition of helium to the TIE-GCM has several feedback effects on the global ${ }_{418}$ structure of the model. Most of these are related to the change in the mean mass, which can become quite small and even approach 4 amu near the top of the model. On levels of 
${ }_{443}$ atmosphere simulated without helium. This modification further couples to the horizontal momentum equations [see Dickinson et al., 1981], increasing horizontal gradients in the

${ }_{445}$ geopotential and resulting in a difference wind pattern that flows away from the winter

${ }_{446}$ helium bulge, as depicted by the vector arrows of Figure 6. This effect generally becomes ${ }_{447}$ noticeable in the upper thermosphere, above $300-400 \mathrm{~km}$, where differences as high as ${ }_{448} \quad 15-20 \mathrm{~m} / \mathrm{s}$ can be attained.

$=$

\section{Summary and Conclusions}

This paper establishes methods for tracking helium abundance self-consistently through- 
${ }_{465}$ boundary to specify a realistic exobase. Using profiles approximated by diffusive equilibrium above the TIE-GCM's upper boundary, we demonstrated that helium can account

${ }_{471}$ the structure of semi-empirical model basis functions [e.g. Sutton et al., 2012]. At a min-

${ }_{472}$ imum, inferring the amplitude of such basis functions would require sufficient coverage of

${ }_{473}$ high-altitude satellite drag measurements, but would be better served by a contemporary

${ }_{474}$ set of mass spectrometer measurements.

${ }_{475}$ The value of helium as a tracer of thermospheric dynamics has been known for some 476 time [see Reber, 1976]. In addition to its ability to diagnose the interplay of circulation ${ }_{477}$ and diffusion in the thermosphere, our new model will enable future studies attempting ${ }_{478}$ to exploit the sensitivity of the helium distribution to otherwise unobservable system ${ }_{479}$ dynamics and inputs. We anticipate that employing helium as a diagnostic tracer${ }_{480}$ e.g. in order to specify or constrain high-latitude energy inputs, solar-driven circulation ${ }_{481}$ pattern strength, and/or sub-grid scale model dynamics - will be beneficial in refining model performance for scientific endeavors as well as operational applications.

\section{Appendix: Time-dependent thermospheric composition for $\mathrm{N}$ components}

In this section, an equation describing the evolution of major species composition in a

${ }_{484}$ log-pressure coordinate frame is derived by combining the species-dependent continuity ${ }_{485}$ and diffusion equations. The derivation closely follows that of Dickinson and Ridley ${ }_{486}[1972]$; however, additional terms describing time dependence, eddy and thermal diffusion 
are included to reflect the current implementation within the TIE-GCM. We also deviate

slightly from their treatment to highlight several equations that are useful in tracking

species-dependent as well as mass-averaged transport. The following definitions are used:

$D_{i j}$ mutual diffusion coefficient of $i$ th and $j$ th components

$g$ gravitational acceleration

$H_{i}$ scale height of $i$ th component $\left[=k T /\left(m_{i} g\right)\right]$

$H$ scale height of mixture $[=k T /(\bar{m} g)]$

$\hat{K}_{E}, K_{E}$ eddy diffusion coefficients

$k$ Boltzmann constant

L differential matrix operator of normalized pressure forces

$m_{i}$ molecular mass of $i$ th component

$\bar{m}$ mean molecular mass $\left[=\left(\sum_{i=1}^{N} n_{i} m_{i}\right) / n\right]$

$n_{i}$ number density of $i$ th component

$n$ total number density $\left[=\sum_{i=1}^{N} n_{i}\right]$

$p_{i} \quad$ partial pressure of $i$ th component $\left[=n_{i} k T\right]$

$p_{0}$ reference pressure

$p$ pressure

$S_{i}$ source or sink for number density of $i$ th component

$\mathrm{s}$ vector containing the first $(N-1)$ components of $m_{i} S_{i} / \rho$

$T$ temperature

V horizontal component of the momentum-weighted mean velocity

$\hat{\mathrm{W}}$ vertical component of the momentum-weighted mean velocity $[=D \hat{z} / D t]$

$w_{i}$ deviation of vertical velocity of $i$ th component from mean velocity

$w_{i}^{\prime}$ contribution to $w_{i}$ from molecular diffusion

$w_{i}^{\prime \prime}$ contribution to $w_{i}$ from eddy diffusion

W vector containing the first $(N-1)$ components of $n_{i} m_{i} w_{i}$

$\mathbf{w}^{\prime}$ vector containing the first $(N-1)$ components of $n_{i} m_{i} w_{i}^{\prime}$

$\mathbf{w}^{\prime \prime}$ vector containing the first $(N-1)$ components of $n_{i} m_{i} w_{i}^{\prime \prime}$

$\hat{z}$ vertical spatial coordinate

$z$ vertical $\log$-pressure coordinate $\left[=\ln \left(p_{0} / p\right)\right]$

$\boldsymbol{\alpha}$ diffusion matrix

$\alpha_{T i}$ thermal diffusion coefficient of $i$ th component

$\theta$ latitude

$\lambda$ longitude

$\nu_{i}$ volume mixing ratio of $i$ th component $\left[=n_{i} / n\right]$

$\rho$ mass density of mixture $\left[=\sum_{i=1}^{N} n_{i} m_{i}\right]$

$\psi_{i}$ relative density of $i$ th component $\left[=n_{i} m_{i} / \rho\right]$

$\Psi$ vector containing the first $(N-1)$ components of $\psi_{i}$

$\omega$ vertical motion relative to $\log$-pressure coordinates $[=D z / D t]$ 


\section{A1. Mass Continuity}

Neglecting horizontal diffusion, each component satisfies the following continuity equa-

491 tion:

$$
\frac{\partial}{\partial \hat{z}}\left(n_{i} m_{i} w_{i}\right)=m_{i} S_{i}-\frac{\partial}{\partial t}\left(n_{i} m_{i}\right)-\nabla \cdot\left(n_{i} m_{i} \mathbf{V}\right)-\frac{\partial}{\partial \hat{z}}\left(n_{i} m_{i} \hat{\mathrm{w}}\right)
$$

The right-hand side of (A1) can be written in terms of the relative densities:

$$
\frac{\partial}{\partial \hat{z}}\left(n_{i} m_{i} w_{i}\right)=m_{i} S_{i}-\left(\frac{\partial}{\partial t}\left(\psi_{i} \rho\right)+\nabla \cdot\left(\psi_{i} \rho \mathbf{V}\right)+\frac{\partial}{\partial \hat{z}}\left(\psi_{i} \rho \hat{\mathrm{w}}\right)\right)
$$

We wish to transform Eq. (A2) from a spatial to a log-pressure vertical coordinate

system under the assumption of hydrostatic equilibrium using the following relationship:

$$
d \hat{z}=H d z
$$

When applying this transformation to partial derivatives with respect to time and horizontal spatial coordinates, the vertical coordinate being held constant must be considered. The following equations, which also require the assumption of hydrostatic equilibrium, are used to complete this transformation [cf. Kasahara, 1974, Eqs. (3.6) and (3.17)]:

$$
\begin{gathered}
\left(\frac{\partial}{\partial t}\right)_{\hat{z}}\left(\psi_{i} \rho\right)=\left(\frac{\partial}{\partial t}\right)_{z}\left(\psi_{i} \rho\right)-\frac{1}{H}\left(\frac{\partial \hat{z}}{\partial t}\right)_{z} \frac{\partial}{\partial z}\left(\psi_{i} \rho\right) \\
\nabla_{\hat{z}} \cdot\left(\psi_{i} \rho \mathbf{V}\right)=\nabla_{z} \cdot\left(\psi_{i} \rho \mathbf{V}\right)-\frac{1}{H}\left(\nabla_{z} \hat{z}\right) \cdot \frac{\partial}{\partial z}\left(\psi_{i} \rho \mathbf{V}\right)
\end{gathered}
$$

where the subscripts $\hat{z}$ and $z$ refer to the vertical coordinate being held constant under partial differentiation. Additionally, the relationship between the spatial and log-pressure 
vertical velocities is as follows [cf. Kasahara, 1974, Eq. (3.12)]:

$$
\hat{\mathrm{w}}=\omega H+\left(\frac{\partial \hat{z}}{\partial t}\right)_{z}+\mathbf{V} \cdot \nabla_{z} \hat{z}
$$

498

Making the appropriate substitutions, noting that the equation of state and our assumption of hydrostatic equilibrium imply:

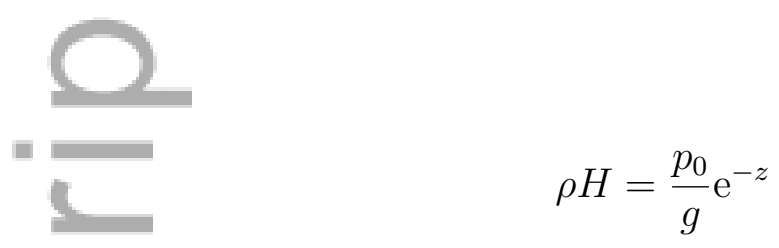

500

and dropping the subscript ' $z$ ' from derivatives taken with respect to time and horizontal spatial coordinates, Eq. A2 becomes:

$$
\frac{\partial}{\partial z}\left(n_{i} m_{i} w_{i}\right)=-\frac{p_{0} \mathrm{e}^{-z}}{g}\left(\frac{\partial \psi_{i}}{\partial t}+\nabla \cdot\left(\psi_{i} \mathbf{V}\right)+\mathrm{e}^{z} \frac{\partial}{\partial z}\left(\psi_{i} \mathrm{e}^{-z} \omega\right)-\frac{m_{i} S_{i}}{\rho}\right)
$$

The definition of $w_{i}$ implies:

$$
\sum_{i=1}^{N} n_{i} m_{i} w_{i}=0
$$

Mass sources are assumed to arise solely from the dissociation of one molecule into

$$
\sum_{i=1}^{N} m_{i} S_{i}=0
$$

Relative densities $\psi_{i}$ are defined so that:

$$
\sum_{i=1}^{N} \psi_{i}=1
$$


By combining (A8) for each component and noting (A9), (A10), and (A11), the continuity equation describing the total fluid in log-pressure coordinates is obtained:

$$
\nabla \cdot \mathbf{V}+\mathrm{e}^{z} \frac{\partial}{\partial z}\left(\mathrm{e}^{-z} \omega\right)=0
$$

Thus, by invoking the assumption of hydrostatic equilibrium and adopting pressure coordinates, the mass flow of the fluid appears incompressible, transforming the mass continuity equation from a prognostic to a diagnostic equation (i.e. no time derivatives appear in the equation).

Using Eq. (A12), the divergence terms of Eq. (A8) can be simplified in favor of advection terms, yielding the following equation:

$$
\widetilde{C} \frac{\partial}{\partial z}\left(n_{i} m_{i} w_{i}\right)=-\frac{p_{0} \mathrm{e}^{-z}}{g}\left(\frac{\partial \psi_{i}}{\partial t}+\mathbf{V} \cdot \nabla \psi_{i}+\omega \frac{\partial \psi_{i}}{\partial z}-\frac{m_{i} S_{i}}{\rho}\right)
$$

Now let $\mathrm{w}$ be the $(N-1)$ vector with components $m_{i} n_{i} w_{i}, \mathbf{s}$ the $(N-1)$ vector with components $m_{i} S_{i} / \rho$, and $\boldsymbol{\Psi}$ the $(N-1)$ vector with elements $\psi_{i}$. Then the first $(N-1)$ equations of (A13) can be written in vector form as:

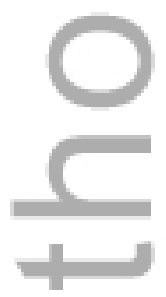

$$
\frac{\partial}{\partial z} \mathbf{w}=-\frac{p_{0}}{g} \mathrm{e}^{-z}\left(\frac{\partial \boldsymbol{\Psi}}{\partial t}+\mathbf{V} \cdot \nabla \boldsymbol{\Psi}+\omega \frac{\partial}{\partial z} \boldsymbol{\Psi}-\mathbf{s}\right)
$$

\section{A2. Molecular and Thermal Diffusion}

With the assumption that the atmosphere is in a state of hydrostatic equilibrium, i.e. $\partial p / \partial \hat{z}=-\rho g$, the equation of motion for the $i$ th component of an $N$-component mixture in the presence of molecular and thermal diffusion [cf. Chapman and Cowling, 1970, Eqs. $(18.2,5)$ and $(18.3,13)]$ can be written: 


$$
\sum_{j \neq i}^{N} \frac{n_{i} n_{j}}{n D_{i j}}\left(w_{j}^{\prime}-w_{i}^{\prime}\right)=n_{i}\left(\frac{1}{p_{i}} \frac{\partial p_{i}}{\partial \hat{z}}+\frac{1}{H_{i}}+\frac{\alpha_{T i}}{T} \frac{\partial T}{\partial \hat{z}}\right)
$$

The pressure force exerted on molecules of the $i$ th component, expressed by the righthand side of (A15), forces these molecules to flow through the rest of the mixture in balance with collisional drags given by the left-hand side.

Noting the partial pressure $p_{i}=p \psi_{i} \bar{m} / m_{i}$, (A15) becomes:

$$
\frac{1}{n} \sum_{j \neq i}^{N}\left[\frac{\psi_{i}}{m_{j} D_{i j}}\left(n_{j} m_{j} w_{j}^{\prime}\right)-\frac{\psi_{j}}{m_{j} D_{i j}}\left(n_{i} m_{i} w_{i}^{\prime}\right)\right]=\left[\frac{\partial}{\partial \hat{z}}-\left(\frac{1}{H}-\frac{1}{H_{i}}-\frac{1}{\bar{m}} \frac{\partial \bar{m}}{\partial \hat{z}}-\frac{\alpha_{T i}}{T} \frac{\partial T}{\partial \hat{z}}\right)\right] \psi_{i}
$$

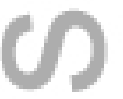

Eqs. (A9) and (A11) — noting that the former applies to ticked quantities as well-are now used to eliminate $w_{N}^{\prime}$ and $\psi_{N}$ from the first $(N-1)$ equations of (A16), giving for the ith component:

$$
\begin{aligned}
& \text { (U) } \\
& \sum_{j=1}^{N-1} \hat{\alpha}_{i j}\left(m_{j} n_{j} w_{j}^{\prime}\right)=\left[\frac{\partial}{\partial \hat{z}}-\left(\frac{1}{H}-\frac{1}{H_{i}}-\frac{1}{\bar{m}} \frac{\partial \bar{m}}{\partial \hat{z}}-\frac{\alpha_{T i}}{T} \frac{\partial T}{\partial \hat{z}}\right)\right] \psi_{i}
\end{aligned}
$$

where

$$
\hat{\alpha}_{i j}= \begin{cases}-\frac{1}{n}\left[\frac{1}{m_{N} D_{i N}}+\sum_{k \neq i}^{N-1}\left(\frac{1}{m_{k} D_{i k}}-\frac{1}{m_{N} D_{i N}}\right) \psi_{k}\right], & j=i \\ \frac{1}{n}\left(\frac{1}{m_{j} D_{i j}}-\frac{1}{m_{N} D_{i N}}\right) \psi_{i}, & j \neq i\end{cases}
$$

and $m_{N}$ refers to the molecular mass of the $N$ th species.

Now let $\hat{\boldsymbol{\alpha}}$ be the $(N-1) \times(N-1)$ matrix with elements $\hat{\alpha}_{i j}$, and $\hat{\mathbf{L}}$ the diagonal matrix of differential operators with elements:

$$
\hat{L}_{i j}=\delta_{i j}\left[\frac{\partial}{\partial \hat{z}}-\left(\frac{1}{H}-\frac{1}{H_{i}}-\frac{1}{\bar{m}} \frac{\partial \bar{m}}{\partial \hat{z}}-\frac{\alpha_{T i}}{T} \frac{\partial T}{\partial \hat{z}}\right)\right]
$$




$$
\mathbf{w}^{\prime}=\hat{\boldsymbol{\alpha}}^{-1} \hat{\mathbf{L}} \Psi
$$

Following Dickinson and Ridley [1972], a nondimensional form of the diffusion matrix

$\hat{\boldsymbol{\alpha}}$ can be derived using a nondimensional parameter $\phi_{i j}$ related to the mutual diffusion

${ }_{535}$ coefficient through:

$$
\phi_{i j}=\frac{m_{N} D}{m_{j} D_{i j}}
$$

where $D$ is a characteristic diffusion coefficient. It is assumed that $D$ varies with pressure ${ }_{537}$ and temperature in the following way:

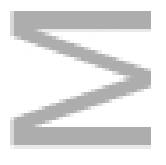

$$
D=D_{0}\left(\frac{p_{00}}{p}\right)\left(\frac{T}{T_{00}}\right)^{1.75}
$$

where $D_{0}=0.2$ is the characteristic diffusion coefficient at S.T.P., $T_{00}=273 \mathrm{~K}, p_{00}=10^{6}$

${ }_{539} \mathrm{~g} /\left(\mathrm{cm} \cdot \mathrm{s}^{2}\right)$

${ }_{540}$ The parameter $\hat{\alpha}_{i j}$ defined by Eq. (A18) is nondimensionalized by the substitution

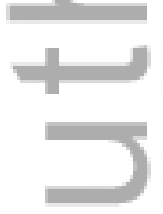

$$
\alpha_{i j}=\left(m_{N} n D\right) \hat{\alpha}_{i j}
$$

${ }_{541}$ where the nondimensional parameter $\alpha_{i j}$ is then

$$
\alpha_{i j}= \begin{cases}-\left[\phi_{i N}+\sum_{k \neq i}^{N-1}\left(\phi_{i k}-\phi_{i N}\right) \psi_{k}\right], & j=i \\ \left(\phi_{i j}-\phi_{i N}\right) \psi_{i}, & j \neq i\end{cases}
$$

D R A F T

August 10, 2015, 8:56pm 
Additionally, Eqs. (A3) and (A7) are again used to transform the vertical coordinate ${ }_{543}$ of the right-hand-side of Eq. (A20) into log-pressure levels, resulting in:

$$
\mathbf{w}^{\prime}=\tau^{-1}\left(\frac{T_{00}}{T}\right)^{0.25} \frac{p_{0} \bar{m}}{m_{N} g} \boldsymbol{\alpha}^{-1} \mathbf{L} \boldsymbol{\Psi}
$$

${ }_{544}$ where

$$
L_{i j}=\delta_{i j}\left[\frac{\partial}{\partial z}-\left(1-\frac{m_{i}}{\bar{m}}-\frac{1}{\bar{m}} \frac{\partial \bar{m}}{\partial z}-\frac{\alpha_{T i}}{T} \frac{\partial T}{\partial z}\right)\right]
$$

${ }_{545} \tau$ is a characteristic diffusion timescale defined by:

$$
\tau=\frac{p_{0}}{p_{00}} \frac{H_{0}^{2}}{D_{0}}
$$

${ }_{546}$ and $H_{0}$ is a characteristic scale height:

$$
H_{0}=\frac{k T_{00}}{m_{N} g}
$$

\section{A3. Eddy Diffusion}

In an atmosphere dominated by a single constituent, as is the case with molecular

Colegrove et al., 1965]:

$$
w_{i}^{\prime \prime}=-\hat{K}_{E} \frac{1}{\nu_{i}} \frac{\partial \nu_{i}}{\partial \hat{z}}
$$


In terms of mass flow rates and mixing ratios, Eq. (A29) becomes:

$$
n_{i} m_{i} w_{i}^{\prime \prime}=-n \bar{m} \hat{K}_{E}\left(\frac{\partial}{\partial \hat{z}}+\frac{1}{\bar{m}} \frac{\partial \bar{m}}{\partial \hat{z}}\right) \psi_{i}
$$

$$
\mathbf{w}^{\prime \prime}=-\frac{p_{0}}{g} K_{E} \mathrm{e}^{-z}\left(\frac{\partial}{\partial z}+\frac{1}{\bar{m}} \frac{\partial \bar{m}}{\partial z}\right) \Psi
$$

${ }_{554}$ where $K_{E} \equiv \hat{K}_{E} / H^{2}$.

\section{A4. Composition Equation}

Setting the total species-dependent mass flux $\mathbf{w}=\mathbf{w}^{\prime}+\mathbf{w}^{\prime \prime}$ and combining Eqs. (A25) and (A31) to eliminate $\mathbf{w}$ from Eq. (A14) yields the composition equation:

$$
\begin{gathered}
\frac{\partial}{\partial z}\left[\tau^{-1}\left(\frac{T_{00}}{T}\right)^{0.25} \frac{\bar{m}}{m_{N}} \boldsymbol{\alpha}^{-1} \mathbf{L} \boldsymbol{\Psi}-K_{E} \mathrm{e}^{-z}\left(\frac{\partial}{\partial z}+\frac{1}{\bar{m}} \frac{\partial \bar{m}}{\partial z}\right) \boldsymbol{\Psi}\right]= \\
\mathrm{e}^{-z}\left(\mathbf{s}-\frac{\partial \boldsymbol{\Psi}}{\partial t}-\mathbf{V} \cdot \nabla \boldsymbol{\Psi}-\omega \frac{\partial}{\partial z} \boldsymbol{\Psi}\right)
\end{gathered}
$$

In the current TIE-GCM implementation, the subscripting order of the major neutral species is as follows: $i=\left\{\mathrm{O}_{2}, \mathrm{O}, \mathrm{He}\right\}$, with $\mathrm{N}_{2}$ chosen to be the $N$ th species due in part to the assumptions stated in Section A3.

Acknowledgments. The authors gratefully acknowledge E. C. Ridley, whose detailed notes regarding the solution of helium as a minor species are maintained by A. Maute of NCAR/HAO. Model simulations and data used throughout this article are freely available by request from ES. This work is supported by AFOSR grant LRIR 13RV09COR to 
${ }_{564}$ the Air Force Research Laboratory, NASA grants NNX10AQ52G and NNX10AE62G to

${ }_{566}$ National Center for Atmospheric Research. NCAR is sponsored by the National Science ${ }_{567}$ Foundation.

\section{References}

${ }_{568}$ Banks, P. M., and G. Kockarts (1973), Aeronomy: Part B, Academic Press. 
0469(1972)029<1557:NSFTCO > 2.0.CO;2.

Dickinson, R. E., E. C. Ridley, and R. G. Roble (1981), A three-dimensional general circulation model of the thermosphere, J. Geophys. Res., 86, 1499-1512, doi: 10.1029/JA086iA03p01499.

Dickinson, R. E., E. C. Ridley, and R. G. Roble (1984), Thermospheric general circulation with coupled dynamics and composition, J. Atmos. Sci., 41, 205-219, doi:10.1175/15200469(1984)041<0205:TGCWCD>2.0.CO;2.

Hagan, M. E., R. G. Roble, and J. Hackney (2001), Migrating thermospheric tides, J. Geophys. Res., 106, 12,739-12,752, doi:10.1029/2000JA000344.

Hays, P. B., R. A. Jones, and M. H. Rees (1973), Auroral heating and the composition of the neutral atmosphere, Planet. Space Sci., 21, 559-573, doi:10.1016/00320633(73)90070-6.

Hedin, A. E. (1987), MSIS-86 thermospheric model, J. Geophys. Res., 92, 4649-4662, doi:10.1029/JA092iA05p04649.

Hedin, A. E. (1991), Extension of the MSIS thermosphere model into the middle and lower atmosphere, J. Geophys. Res., 96, 1159-1172, doi:10.1029/90JA02125.

Hedin, A. E., and G. R. Carignan (1985), Morphology of thermospheric composition variations in the quiet polar thermosphere from Dynamics Explorer measurements, $J$. Geophys. Res., 90, 5269-5277, doi:10.1029/JA090iA06p05269.

Hedin, A.E., H. G. Mayr, C. A. Reber, N. W. Spencer, and G. R. Carignan (1974), Empirical model of global thermospheric temperature and composition based on data from the Ogo 6 quadrupole mass spectrometer, J. Geophys. Res., 79, 215, doi: 10.1029/JA079i001p00215. 
Heelis, R. A., J. K. Lowell, and R. W. Spiro (1982), A model of the highlatitude ionospheric convection pattern, J. Geophys. Res., 8\%, 6339-6345, doi: 10.1029/JA087iA08p06339.

Hodges, R. R., Jr. (1970), Vertical transport of minor constituents in the lower thermosphere by nonlinear processes of gravity waves, J. Geophys. Res., 75, 4842-4848, doi:10.1029/JA075i025p04842.

Hodges, R. R., Jr. (1973), Differential equation of exospheric lateral transport and its application to terrestrial hydrogen, J. Geophys. Res., 78, 7340-7346, doi: 10.1029/JA078i031p07340.

Hodges, R. R., Jr., and F. S. Johnson (1968), Lateral transport in planetary exospheres, J. Geophys. Res., 73, 7307, doi:10.1029/JA073i023p07307.

Johnson, F. S., and B. Gottlieb (1970), Eddy mixing and circulation at ionospheric levels, Planet. Space Sci., 18, 1707-1718, doi:10.1016/0032-0633(70)90004-8.

Kasahara, A. (1974), Various Vertical Coordinate Systems Used for Numerical Weather Prediction, Monthly Weather Review, 102, 509, doi:10.1175/15200493(1974)102<0509:VVCSUF>2.0.CO;2.

Kasprzak, W.T. (1969), Evidence for a helium flux in the lower thermosphere, J. Geophys. Res., 74, 894-896, doi:10.1029/JA074i003p00894.

Keating, G. M., and E. J. Prior (1968), The winter helium bulge, in Space Research VIII, edited by A. P. Mitra, L. G. Jacchia, and W. S. Newman, p. 982.

Keating, G. M., J. A. Mullins, and E. J. Prior (1970), The polar exosphere near solar maximum., in Space Research X, edited by T. M. Donahue, P. A. Smith, and L. Thomas, pp. 439-449. 
Keating, G. M., E. J. Prior, D. S. McDougal, and J. I. Nicholson (1974), Critical evaluation of the OGO 6 helium model, in Space Research XV, edited by M. J. Rycroft, pp. 273278.

Keeling, C. D., and T. P. Whorf (2005), Atmospheric co2 records from sites in the sio air sampling network, Trends: A Compendium of Data on Global Change. Carbon Dioxide Information Analysis Center, Oak Ridge National Laboratory, Oak Ridge, TN.

Kockarts, G. (1972), Distribution of hydrogen and helium in the upper atmosphere., J. Atmos. Terr. Phys., 34, 1729-1743, doi:10.1016/0021-9169(72)90032-3.

Kockarts, G. (1973), Helium in the Terrestrial Atmosphere, Space Science Reviews, 14, 723-757, doi:10.1007/BF00224775.

Lettau, H.'(1951), Diffusion in the upper atmosphere, in Compendium of Meteorology, pp. 320-333, Amer. Meteor. Soc.

Liu, X., J. P. Thayer, A. Burns, W. Wang, and E. K. Sutton (2014a), Altitude variations in the thermosphere mass density response to geomagnetic activity during the recent solar minimum, J. Geophys. Res., 119, 2160-2177, doi:10.1002/2013JA019453.

Liu, X., W. Wang, J. P. Thayer, A. Burns, E. K. Sutton, S. C. Solomon, L. Qian, and G. Lucas (2014b), The winter helium bulge revisited, Geophys. Res. Lett., doi: 10.1002/2014GL061471.

Mauersberger, K., D. C. Kayser, W. E. Potter, and A. O. Nier (1976a), Seasonal variation of neutral thermospheric constituents in the Northern Hemisphere, J. Geophys. Res., 81, 7-11, doi:10.1029/JA081i001p00007.

Mauersberger, K., W. E. Potter, and D. C. Kayser (1976b), A direct measurement of the winter helium bulge, Geophys. Res. Lett., 3, 269-271, doi:10.1029/GL003i005p00269. 
${ }_{654}$ Mayr, H. G., and H. Volland (1972), Theoretical model for the latitude dependence of the thermospheric annual and semiannual variations, J. Geophys. Res., 77, 6774, doi: 10.1029/JA077i034p06774.

Mayr, H. G., and H. Volland (1973), A two-component model of the diurnal variations in the thermospheric composition, Journal of Atmospheric and Terrestrial Physics, 35, 669, doi:10.1016/0021-9169(73)90198-0.

Mayr, H. G., A. E. Hedin, C. A. Reber, and G. R. Carignan (1974), Global characteristics in the diurnal variations of the thermospheric temperature and composition, J. Geophys. Res., 79,619, doi:10.1029/JA079i004p00619.

Mayr, H. G., I. Harris, and N. W. Spencer (1978), Some properties of upper atmosphere dynamics, Reviews of Geophysics and Space Physics, 16, 539-565, doi: 10.1029/RG016i004p00539.

Newton, G. P., D. T. Pelz, and W. T. Kasprzak (1973), Equatorial thermospheric composition and its variations, in Space Research XIII, edited by M. J. Rycroft and S. K. Runcorn, pp. 287-290.

Nicolet, M. (1961), Helium, an Important Constituent in the Lower Exosphere, J. Geophys. Res., 66, 2263-2264, doi:10.1029/JZ066i007p02263.

Picone, J. M., A. E. Hedin, D. P. Drob, and A. C. Aikin (2002), NRLMSISE-00 empirical model of the atmosphere: Statistical comparisons and scientific issues, J. Geophys. Res., 107, 1468, doi:10.1029/2002JA009430.

Qian, L., S. C. Solomon, and T. J. Kane (2009), Seasonal variation of thermospheric density and composition, J. Geophys. Res., 114, A01312, doi:10.1029/2008JA013643. 
Qian, L., A. G. Burns, S. C. Solomon, and W. Wang (2013), Annual/semiannual variation of the ionosphere, Geophys. Res. Lett., 40, 1928-1933, doi:10.1002/grl.50448.

Reber, C. A. (1976), Dynamical effects in the distribution of helium in the thermosphere, J. Atmos. Terr. Phys., 38, 829-840, doi:10.1016/0021-9169(76)90023-4.

Reber, C.A., and P. B. Hays (1973), Thermospheric wind effects on the distribution of helium and argon in the Earth's upper atmosphere, J. Geophys. Res., 78, 2977, doi: 10.1029/JA078i016p02977.

Reber, C. A., and M. Nicolet (1965), Investigation of the major constituents of the AprilMay 1963 heterosphere by the Explorer XVII satellite, Planet. Space Sci., 13, 617-646, doi:10.1016/0032-0633(65)90043-7.

Reber, C. A., D. N. Harpold, R. Horowitz, and A. E. Hedin (1971), Horizontal distribution of helium in the Earth's upper atmosphere, J. Geophys. Res., 76, 1845, doi: 10.1029/JA076i007p01845.

Reber, C.A., A. E. Hedin, and S. Chandra (1973), Equatorial phenomena in neutral thermo-spheric composition, J. Atmos. Terr. Phys., 35, 1223, doi:10.1016/00219169(73)90019-6.

Reber, C. A., A. E. Hedin, D. T. Pelz, L. H. Brace, and W. E. Potter (1975), Phase and amplitude relationships of wave structure observed in the lower thermosphere, $J$. Geophys. Res., 80, 4576-4580, doi:10.1029/JA080i034p04576.

Richards, P. G., J. A. Fennelly, and D. G. Torr (1994), EUVAC: A solar EUV flux model for aeronomic calculations, J. Geophys. Res., , 99, 8981-8992, doi:10.1029/94JA00518. Richmond, A. D., E. C. Ridley, and R. G. Roble (1992), A thermosphere/ionosphere general circulation model with coupled electrodynamics, Geophys. Res. Lett., 19, 601- 
604, doi:10.1029/92GL00401.

Roble, R. G., and E. C. Ridley (1987), An auroral model for the NCAR thermospheric general circulation model (TGCM), Annales Geophysicae, 5, 369-382.

Roble, R. G., and E. C. Ridley (1994), A thermosphere-ionosphere-mesosphereelectrodynamics general circulation model (time-GCM): Equinox solar cycle minimum simulations (30-500 km), Geophys. Res. Lett., 21, 417-420, doi:10.1029/93GL03391.

Roble, R. G., E. C. Ridley, A. D. Richmond, and R. E. Dickinson (1988), A coupled thermosphere/ionosphere general circulation model, Geophys. Res. Lett., 15, 1325-1328, doi:10.1029/GL015i012p01325.

Schunk, R. W., and A. F. Nagy (2004), Ionospheres, Cambridge Atmospheric and Space Science Series.

Solomon, S. C., and L. Qian (2005), Solar extreme-ultraviolet irradiance for general circulation models, Journal of Geophysical Research (Space Physics), 110, A10306, doi: 10.1029/2005JA011160.

Solomon, S. C., L. Qian, L. V. Didkovsky, R. A. Viereck, and T. N. Woods (2011), Causes of low thermospheric density during the 2007-2009 solar minimum, J. Geophys. Res., 116, A00H07, doi:10.1029/2011JA016508.

Solomon, S. C., A. G. Burns, B. A. Emery, M. G. Mlynczak, L. Qian, W. Wang, D. R. Weimer, and M. Wiltberger (2012), Modeling studies of the impact of high-speed streams and co-rotating interaction regions on the thermosphere-ionosphere, J. Geophys. Res., 117, A00L11, doi:10.1029/2011JA017417.

Sutton, E.K. (2011), Accelerometer-derived atmospheric density from the CHAMP and GRACE satellites, AFRL Tech. Rep., DTIC\# ADA537198. 
${ }_{722}$ Sutton, E. K., S. B. Cable, C. S. Lin, L. Qian, and D. R. Weimer (2012), Thermo-

spheric basis functions for improved dynamic calibration of semi-empirical models, Space Weather, 10(10), S10001, doi:10.1029/2012SW000827.

Swarztrauber, P. (1979), On the spectral approximation of discrete scalar and vector functions on the sphere, SIAM Journal on Numerical Analysis, 16(6), 934-949, doi: $10.1137 / 0716069$.

Thayer, J. P., X. Liu, J. Lei, M. Pilinski, and A. G. Burns (2012), The impact of helium on thermosphere mass density response to geomagnetic activity during the recent solar minimum, Journal of Geophysical Research (Space Physics), 11\%, A07315, doi: 10.1029/2012JA017832.

Viereck, R. A., L. E. Floyd, P. C. Crane, T. N. Woods, B. G. Knapp, G. Rottman, M. Weber, L. C. Puga, and M. T. Deland (2004), A composite Mg II index spanning from 1978 to 2003, Space Weather, 2, 10005, doi:10.1002/2004SW000084.

Woods, T. N., and G. J. Rottman (2002), Solar Ultraviolet Variability Over Time Periods of Aeronomic Interest, Washington DC American Geophysical Union Geophysical Monograph Series, 130, 221.

Zhang, Y., and L. J. Paxton (2008), An empirical Kp-dependent global auroral model based on TIMED/GUVI FUV data, J. Atmos. Sol.-Terr. Phys., 70, 1231-1242, doi: 10.1016/j.jastp.2008.03.008.

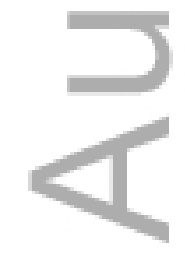


Table 1. Mütual diffusion coefficients for helium with major species ${ }^{1}$.

\begin{tabular}{|c|c|c|}
\hline$i-j$ & $a$ & $s$ \\
\hline $\mathrm{He}-\mathrm{O}_{2}$ & 0.649 & 1.710 \\
\hline $\mathrm{He}-\mathrm{O}$ & 0.866 & 1.749 \\
\hline $\mathrm{He}-\mathrm{N}_{2}$ & 0.622 & 1.718 \\
\hline
\end{tabular}

1973, table 15.1$]$.

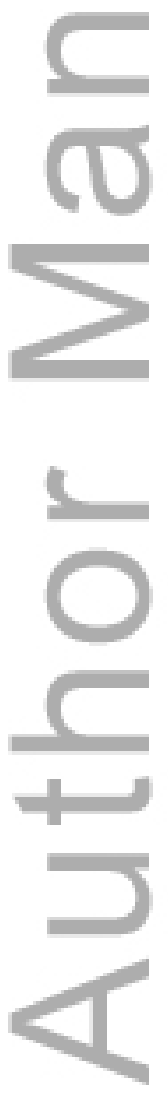




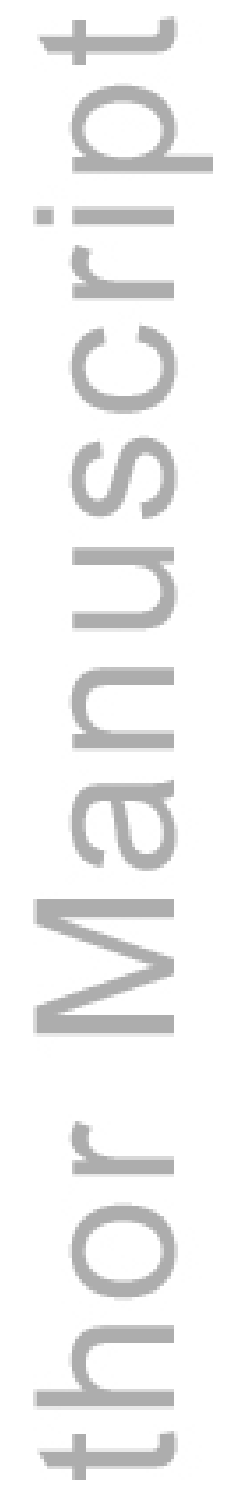

Figure 1. MSIS mass mixing ratios for $\mathrm{O}_{2}$ (blue), $\mathrm{O}$ (green), $\mathrm{N}_{2}$ (red), and $\mathrm{He}$ (cyan) calculated on the vertical log-pressure scale in the vicinity of the winter helium bulge for solar maximum (solid lines/black altitude labels, 21 Dec., 2000) and minimum (dashed lines/grey altitude labels, 21 Dec., 2008) conditions. 


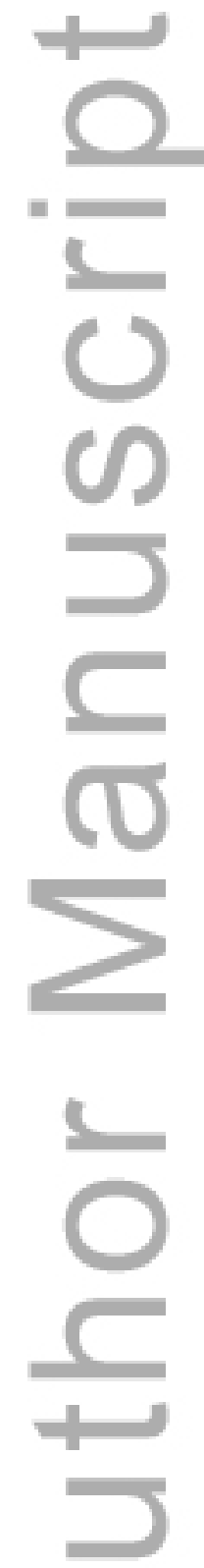

Figure 2. Global distribution of helium number densities at $250 \mathrm{~km}$ altitude during each season for solar minimum conditions (2008), as calculated by TIE-GCM. Equinox plots (left) share a common color scale, as do solstice plots (right). 


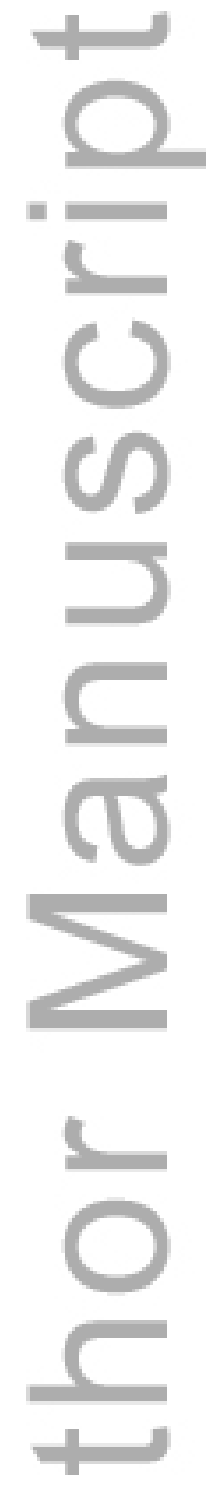

Figure 3. Global distribution of helium number densities at $250 \mathrm{~km}$ altitude during each season for solar minimum conditions (2008), as calculated by MSIS. Equinox plots (left) share a common color scale, as do solstice plots (right); these are distinct from the color scales of Figure 2. 

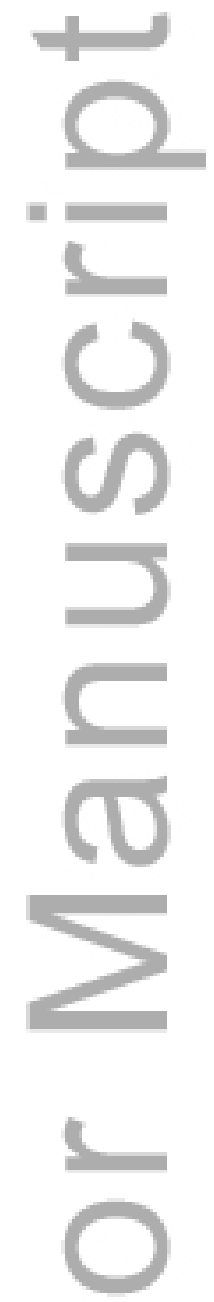

Figure 4. Vertical profile of the winter-to-summer helium bulge ratio during solar minimum June (black, June 21, 2008) and December (grey, Dec. 21, 2008) solstice conditions. The profiles represent the daily average of the ratio of maximum-to-minimum helium number densities taken along each meridian, roughly approximating the sampling of a polar orbiting satellite (see text for a detailed explanation). Error bars indicate the standard deviation of values over the course of a day. 


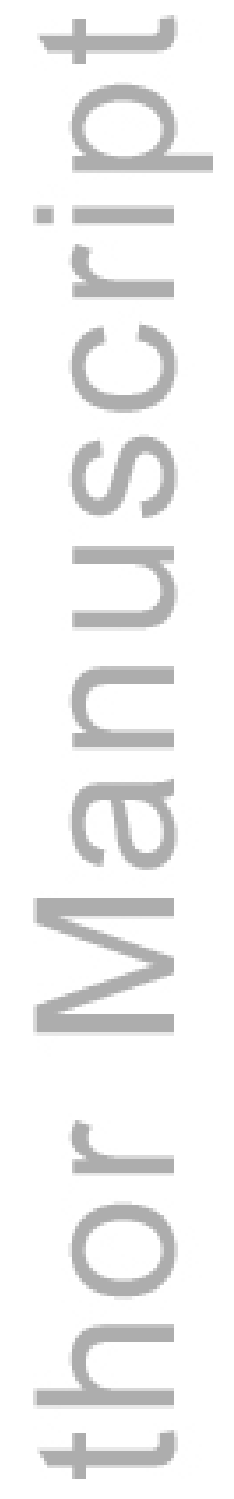

Figure 5. Percent increase in the total mass density at a fixed altitude of $415 \mathrm{~km}$ resulting from the inclusion of helium in TIE-GCM during each season for solar minimum conditions (2008). Equinox plots (left) share a common color scale, as do solstice plots (right). 


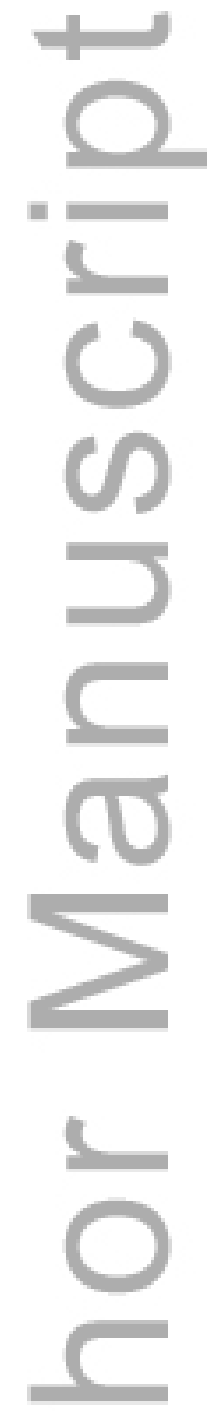

Figure 6. Difference in the geopotential height (color contours) and the horizontal wind field (vectors) on a level of constant pressure near the upper model boundary $(z=+6.75)$ resulting from the inclusion of helium in TIE-GCM during each season for solar minimum conditions (2008). Equinox plots (left) share common color and vector scales, as do solstice plots (right). 


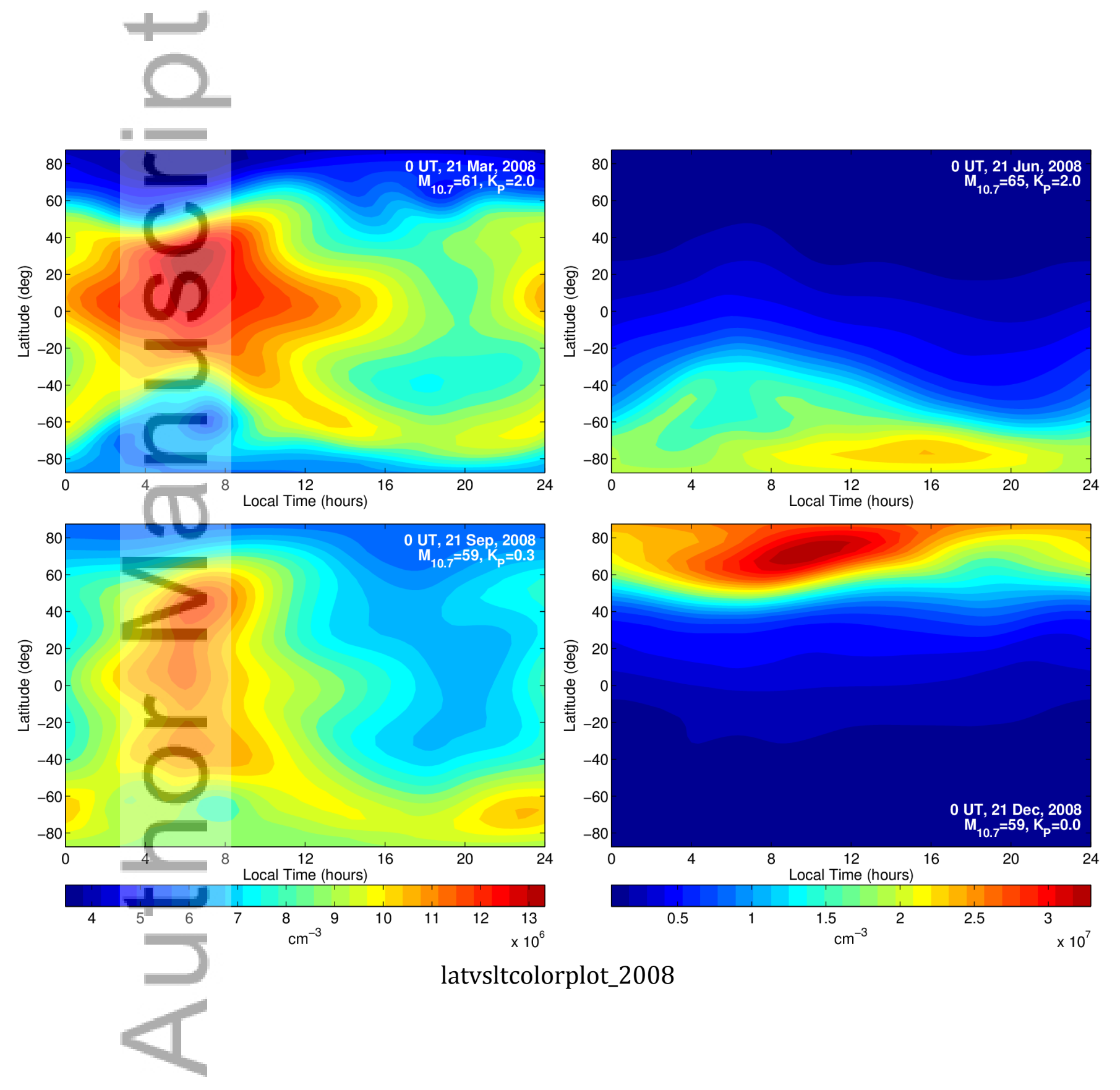

This article is protected by copyright. All rights reserved. 

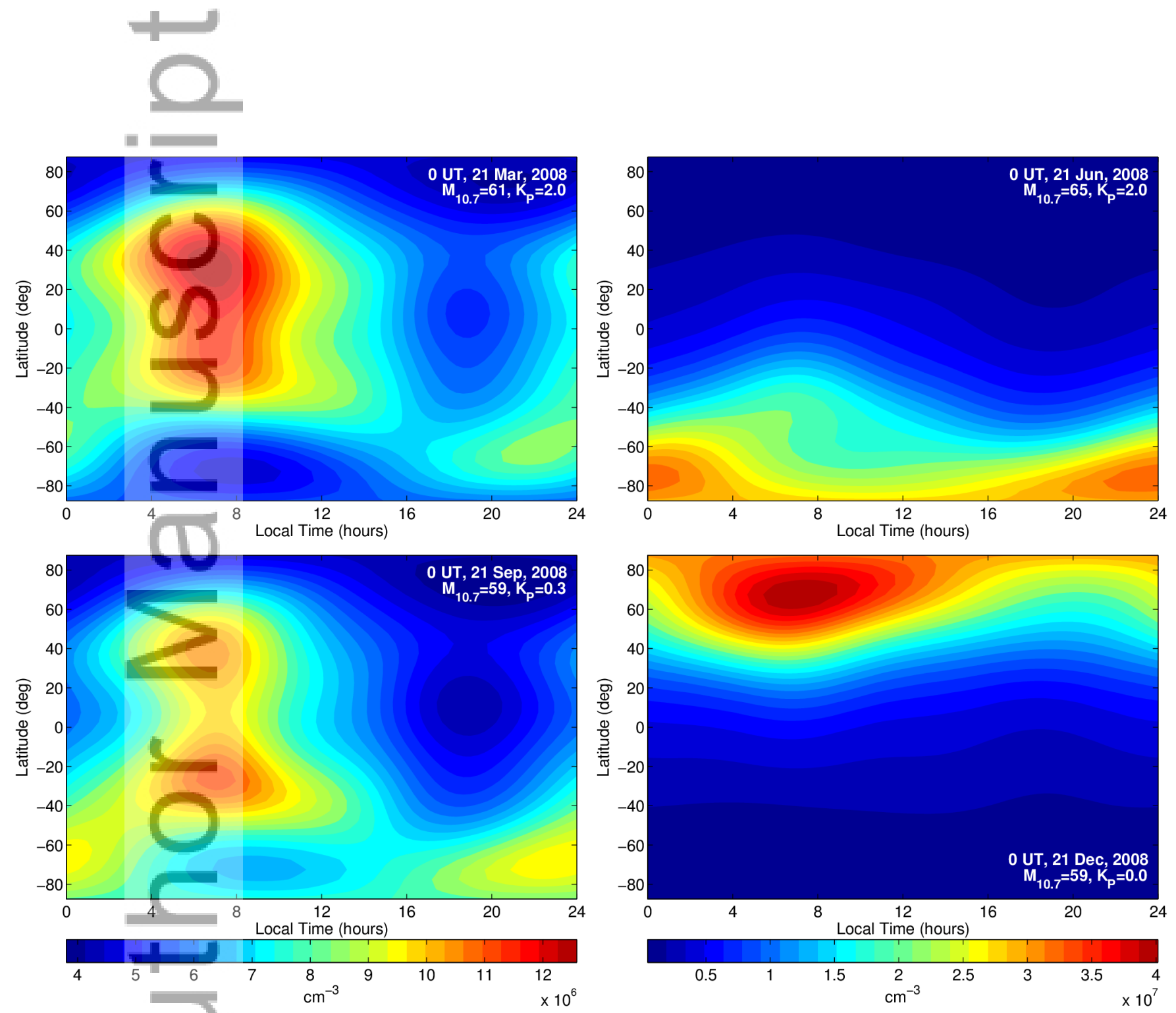

latvsltcolorplot_msis_2008

This article is protected by copyright. All rights reserved. 

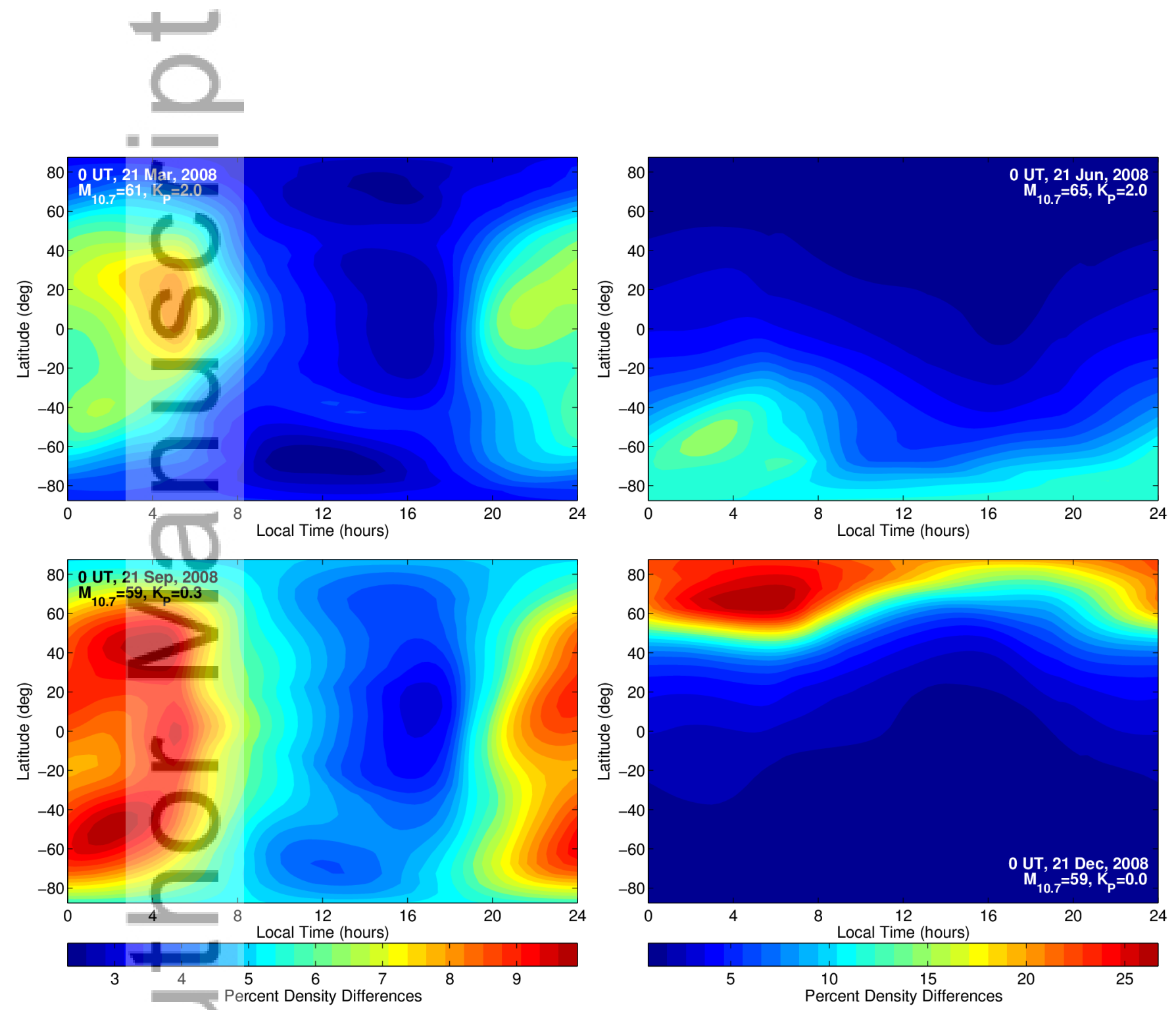

latvsltcolorplot_rho415km_2008

This article is protected by copyright. All rights reserved. 

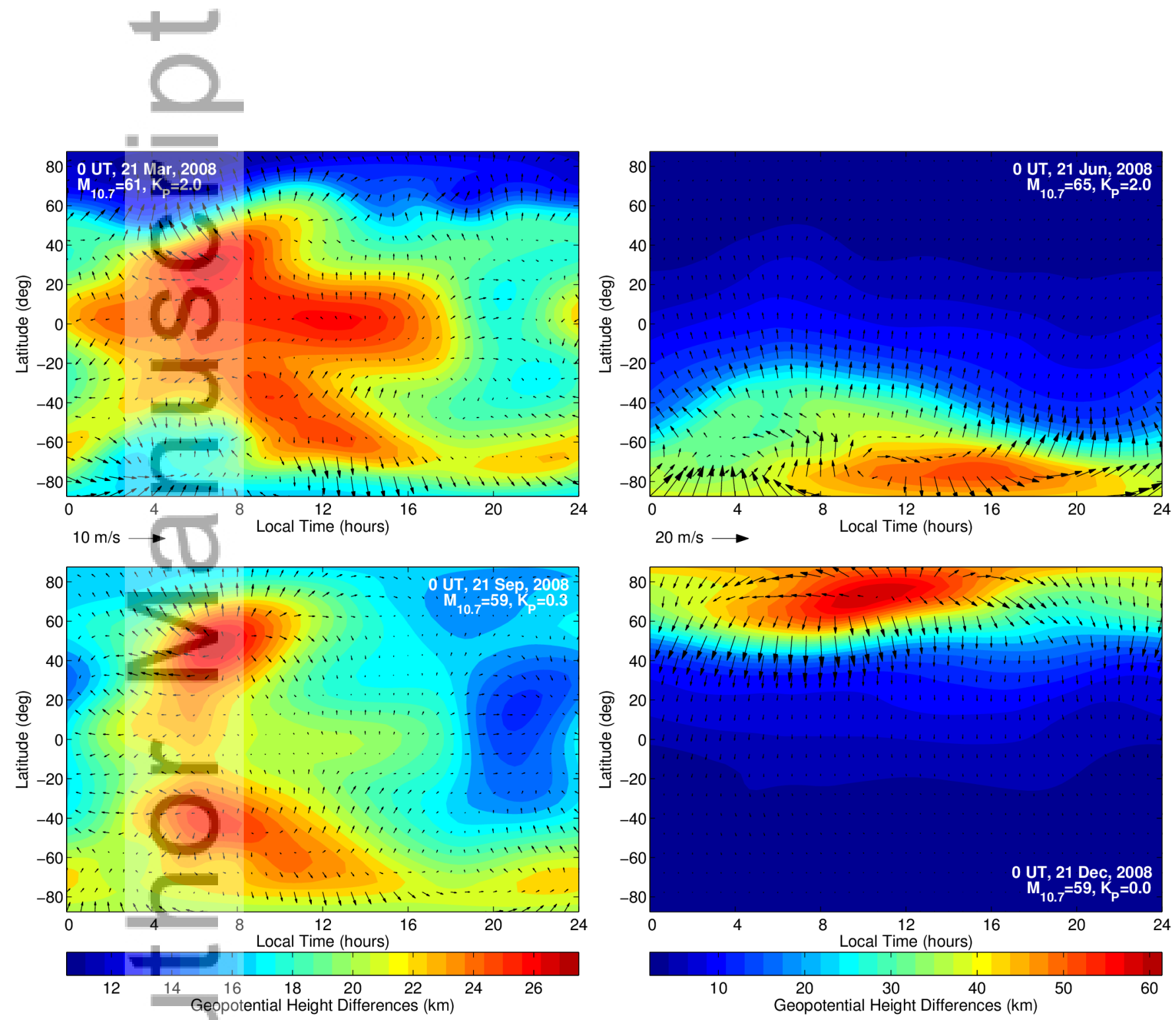

latvsltcolorplot_windgeopot_lev28_2008 


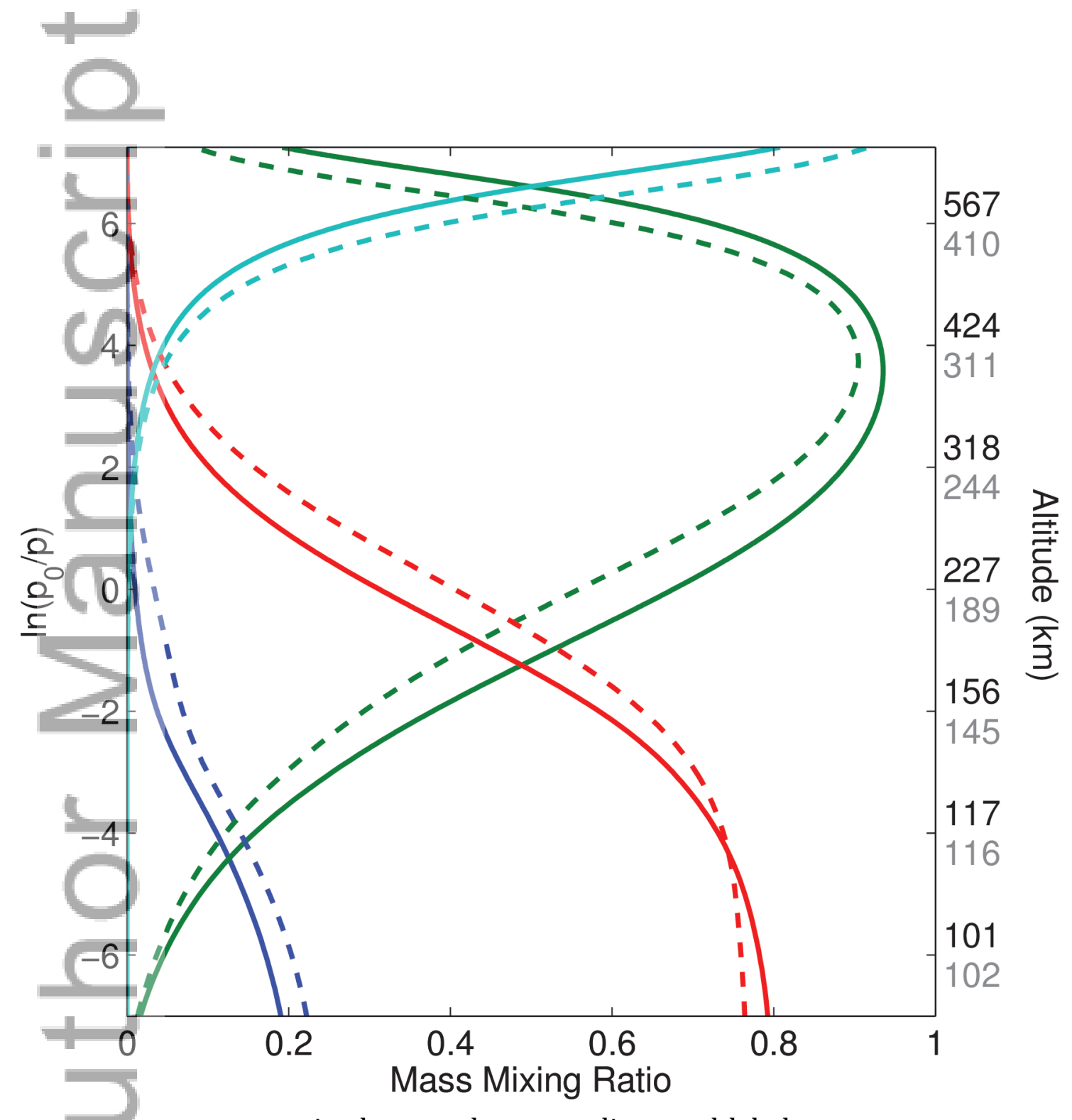

msis_alternate_logp_coordinates_altlabels 


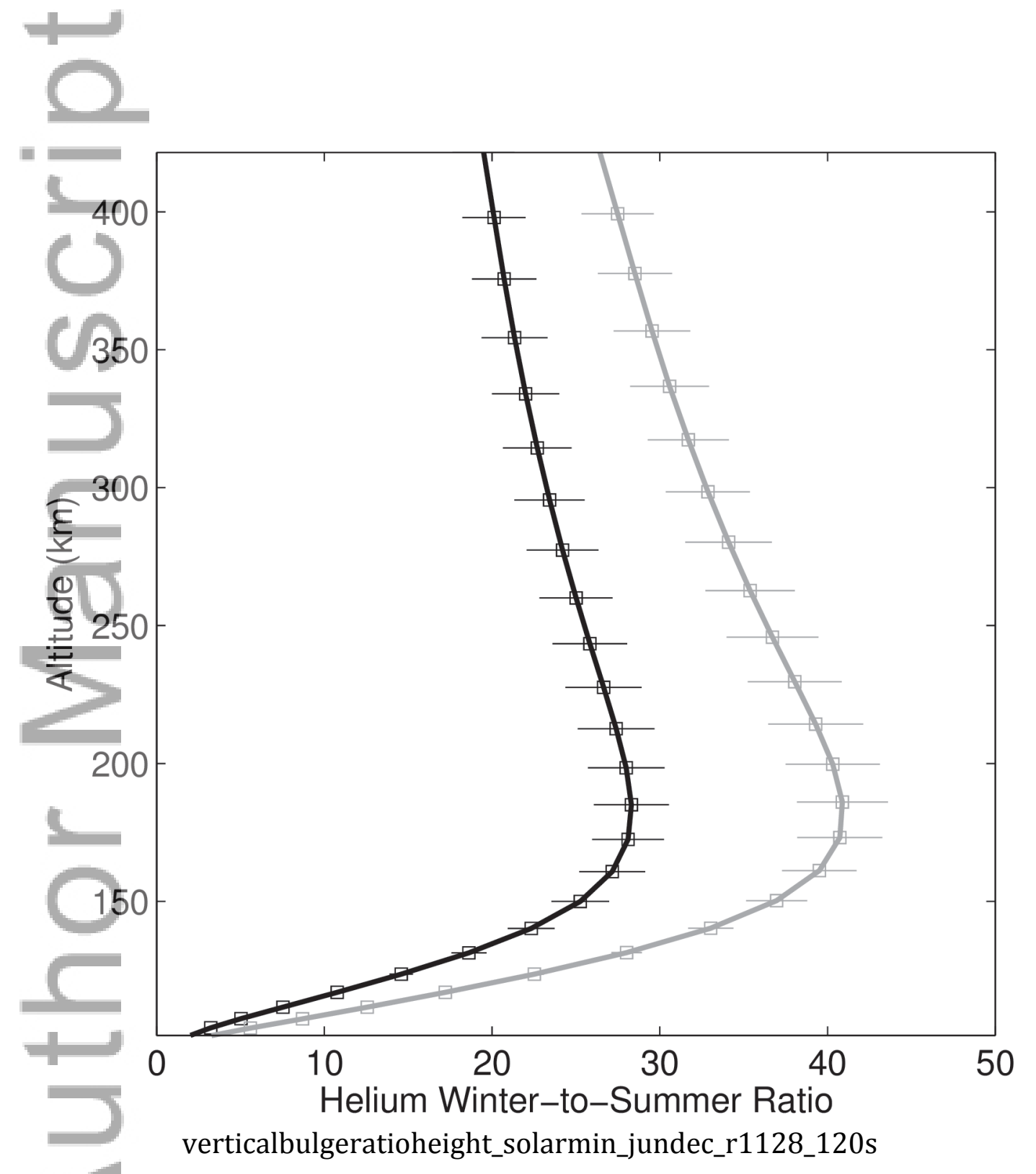

This article is protected by copyright. All rights reserved. 